Volume 8, No.1.6, 2019

International Journal of Advanced Trends in Computer Science and Engineering

Available Online at http://www.warse.org/IJATCSE/static/pdf/file/ijatcse3981.62019.pdf

https://doi.org/10.30534/ijatcse/2019/3981.62019

\title{
Stabilizing Roll, Pitch and Yaw Angles for Attitude Control System (ACS) using Direct Torque Control (DTC)
}

\author{
AnandKumar Deva Dass ${ }^{1}$, Hilmi Sanusi ${ }^{1}$ \\ Anuar M. Muad ${ }^{2}$ \\ ${ }^{1}$ Centre of Advanced Electronic and Communication Engineering (PAKET) \\ ${ }^{2}$ Centre for Integrated Systems Engineering and Advaced Technologies (INTEGRA) \\ Faculty of Engineering \& Built Environment, \\ Universiti Kebangsaan Malaysia \\ anandkumar@siswa.ukm.edu.my \\ hilmisanusi@ukm.edu.my \\ anuar_muad@ukm.edu.my
}

\begin{abstract}
This paper presents the development of a Direct Torque Control (DTC) as the controller in Attitude Determination and Control System (ADCS) of Satellite. The attitude determination is a process of determining the offset angle between the orbit reference angle and body angle. The Attitude control is a process to reorient the satellite in the desired attitude by producing additional torque to the body of the satellite. The controller is constructed using Proportional-Integrator-Derivatives (PID) and followed by a DTC on the actuator. The demand torque to the actuator is computed by PID from the error between the desired attitude and estimated attitude. The DTC estimates the error between the torque demand and actual torque generated by the actuator, and then the correct torque is generated by the actuator. The simulation is verified through closed-loop simulation as presented in the result. The simulation shows attitude control of a satellite shows a promising result by using a DTC.
\end{abstract}

Key words : Attitude determination, Attitude control, Triad Algorithm, PID, Direct Torque Control

\section{INTRODUCTION}

The Attitude Determination and Control System (ADCS) is a crucial subsystem of a spacecraft. It provides the pointing accuracy and stabilization, and reorientation of the satellite in a given direction which allows a spacecraft to successfully perform its mission objectives. To support the satellite mission, the ADCS is required to provide the in-orbit attitude control and determination functions, which are the Attitude Control System (ACS) and Attitude Determination System (ADS). After the separation of the satellite from the launcher, the ADCS will also operate and record the pertinent data. The attitude determination function is to facilitate the estimated attitude information, while the attitude control function includes the stabilization and detumbling control of the satellite to the prerequisite value. The ADS utilizes the data from various sensors such as gyroscope, accelerometer, sun sensor and magnetometer $[3,7,8,12,13]$ to obtain the attitude of the satellite-based on the instantaneous method (such as TRIAD or QUEST) or recursive method (such as RE-QUEST or Kalman Filter). As for the ACS, there are few actuators such as reaction wheel, momentum wheels, magnetic torquer, thruster and others that can produce torque to change the orientation of the satellite. In order to produce the correct amount of torque, a closed-loop system is employed [19] and certain control laws, from as simple as a PID controller to a complex method is used to obtain the desired roll, pitch, and yaw. A reaction wheel consists of a Permanent Magnet Direct Current (PM-DC) with some angular mass constructed as an actuator and PID as the control law [18] are widely been used in the ACS.

However, this technique has a significant amount of inaccuracies in the attitude due to variation in the torque generated by actuators. Direct Torque Control (DTC) can be considered to be implemented for better control of the torque. Direct Torque Control is one of the two types of instantaneous electromagnetic torque-controlled $\mathrm{AC}$ drives used in the high-performance applications [11] due to stator flux control in the stator fixed reference frame using direct control of the inverter switching. Due to the absence of coordinate transformation between a stationary frame and synchronous frame and PI regulators, the DTC algorithm is much simpler. The implementation of the DTC has been established about many years ago and proven to be one of the effective ways to control the induction machine, but the usage of DTC in the ADCS is still not yet explored.

This paper presents the development of a Direct Torque Control (DTC) as the controller in the Attitude Determination and Control System (ADCS) of Satellite. The results have shown that the possibility of implementing DTC to control the speed of a reaction wheel.

\section{METHODOLOGY}

This ADCS system was developed using 4 main components which are the environmental model, satellite model, Attitude determination and Attitude control mode. Each of these mathematical models works collectively in computing the satellite's attitude and controlling it. 


\subsection{Environmental model}

The environmental model serves as the environment in which the satellite operates. This model consists of the sun, basic orbit propagator, and Earth magnetic field model. The orbit propagator generates the satellite's orbit based on the time increment and the initial orbital elements and computes the position and velocity vector of the satellite. Sun model generates the sun reference vector relative to the earth and earth magnetic field model calculates the magnetic field vector from the position and velocity of the satellite.

\subsection{Satellite model}

This model describes the satellite's body, kinetic and kinematics of the satellite about its center mass. For the satellite's rigid model, a principal moment of inertia is evaluated to ensure the stability of the dynamic system. To achieve a stable dynamic system, the principal moment of inertia $I_{x}, I_{y}, I_{z}$ must satisfy the following conditions:

$$
\begin{aligned}
& K_{x}=\frac{\left(I_{y}-I_{z}\right)}{I_{x}}, K_{y}=\frac{\left(I_{x}-I_{z}\right)}{I_{y}}, \quad K_{z}=\frac{\left(I_{y}-I_{x}\right)}{I_{z}} \\
& K_{x} K_{z}>0 \\
& 1+3 K_{x}+K_{x} K_{z}>0 \\
& \left(1+3 K_{x}+K_{x} K_{z}\right)^{2}-16 K_{x} K_{z}>0
\end{aligned}
$$

The dynamic equations of the satellite are as follows:

$$
\begin{aligned}
& I_{x} \dot{\varphi}=-\omega_{0}^{2}\left(I_{y}-I_{z}\right) \varphi+\omega_{0}\left(I_{x}-I_{z}+I_{z}\right) \dot{\psi} \\
& I_{y} \theta=0 \\
& I_{z} \dot{\psi}=-\omega_{0}^{2}\left(I_{y}-I_{x}\right) \psi+\omega_{0}\left(I_{x}-I_{z}+I_{z}\right) \dot{\varphi}
\end{aligned}
$$

The dynamic equations after the addition of the gravity gradient torque [3]

$$
\begin{aligned}
& I_{x} \dot{\varphi}=-4 \omega_{0}^{2}\left(I_{y}-I_{z}\right) \varphi+\omega_{0}\left(I_{x}-I_{z}+I_{z}\right) \dot{\psi} \\
& I_{y} \theta=-3 \omega_{0}^{2}\left(I_{x}-I_{y}\right) \dot{\theta} \\
& I_{z} \dot{\psi}=-\omega_{0}^{2}\left(I_{y}-I_{x}\right) \psi+\omega_{0}\left(I_{x}-I_{z}+I_{z}\right) \dot{\varphi}
\end{aligned}
$$

The complete linearized dynamic motion of satellite with input torque

$$
\begin{aligned}
& I_{x} \dot{\varphi}=-4 \omega_{0}^{2}\left(I_{y}-I_{z}\right) \varphi+\omega_{0}\left(I_{x}-I_{z}+I_{z}\right) \dot{\psi}+\tau_{x} \\
& I_{y} \theta=-3 \omega_{0}^{2}\left(I_{x}-I_{y}\right) \dot{\theta}+\tau_{y} \\
& I_{z} \dot{\psi}=-\omega_{0}^{2}\left(I_{y}-I_{x}\right) \psi+\omega_{0}\left(I_{x}-I_{z}+I_{z}\right) \dot{\varphi}+\tau_{z}
\end{aligned}
$$

\subsection{Attitude Determination}

Attitude determination of this ADCS system uses a deterministic method using the TRIAD algorithm. The solution is based on two vector observations given in two different coordinate systems. TRIAD only accommodates two vector observations at a one-time instance. The simplicity of the solution makes the TRIAD method interesting for onboard implementations [7]. Considering this assumption, body vectors are assumed $b_{1}, b_{2}$ and inertial frame are assumed to be $i_{1}, i_{2}$. Based on this vector measurements, the attitude matrix is constructed using the TRIAD algorithm as following:

$$
\begin{array}{ll}
t_{1 b}=\frac{b_{1}}{\left|b_{1}\right|}, & t_{1 i}=\frac{i_{1}}{\left|i_{1}\right|} \\
t_{2 b}=\frac{b_{1} \times b_{2}}{\left|b_{1} \times b_{2}\right|}, & t_{2 i}=\frac{i_{1} \times i_{2}}{\left|i_{1} \times i_{2}\right|} \\
t_{3 b}=t_{1 b} \times t_{2 b} & t_{3 i}=t_{1 i} \times t_{2 i} \\
A_{\text {triad }}=\left[t_{1 b} t_{2 b} t_{3 b}\right] \cdot\left[t_{1 i} t_{2 i} t_{3 i}\right]
\end{array}
$$

This attitude matrix gives the estimated attitude of the satellite in terms of the rotation matrix. Then the Euler angles describing attitude can be extracted out from the rotation matrix. In this system, the body vector measurements are computed using the attitude matrix rather than the sun sensor and magnetometer actual readings.

\subsection{Attitude control}

Attitude control in this system is used to reorient the satellite based on the desired attitude. The attitude control of the ADCS system can be achieved by using two methods active and passive control. Initially, the satellite experiences the detumbling mode during which the roll, pitch, and yaw angle increase exponentially collectively known as Euler's angle. At this phase, the passive control using gravity gradient torque is introduced to the system to achieve the stabilization mode. The gravity gradient torque for this system is fixed by assuming the orbit is circular with small-angle approximation. The gravity gradient torque equals to:

$$
\begin{aligned}
\tau_{x} & =3 \omega_{0}^{2}\left(I_{z}-I_{y}\right) \dot{\varphi} \\
\tau_{y} & =3 \omega_{0}^{2}\left(I_{z}-I_{x}\right) \dot{\theta} \\
\tau_{z} & =0
\end{aligned}
$$

Once the stabilization mode has been reached, active control is implemented using a PID controller and Direct Torque Control. The error between the desired and estimated attitude raised to compute the required input torque:

$$
u(t)=K_{P}(e(t) d t)+1 / T_{I} \int e(t) d t+T_{D} \frac{d e(t)}{d t}
$$

where,

$$
\tau(t)=u(t)
$$

This is the input torque required to reorient the satellite. This input torque is feed into the Direct Torque Control to generate torque to move the satellite. The Direct Torque Control is used in this system to correct the conventional active attitude control devices such as magnetic actuators and reaction wheel. This is performed since the Direct Torque Control has a much better inner loop design and corrects the torque generated by the actuators based on stator flux control. 


\subsection{Actuator Model}

The actuator used in this ADCS system is Magnetic Torquer. The torque generated by the Magnetic Torquer is given by:

$$
\tau=m \times B_{e}
$$

Where $\tau$ is the torque generated, $m$ is the magnetic dipole moment and $B_{e}$ is the earth magnetic field. The magnetic dipole moment determined by using:

$$
m=N I A
$$

Where $N$ is the number of coils, $I$ current flowing through the coil and $A$ is area span of coil.

\section{RESULTS AND DISCUSSION}

The overall ADCS system is built using the Simulink model in the Matlab. The Environmental and Attitude determination model were built using the Matlab function. The environmental model and attitude determination model compute the estimated attitude based on the input data which are time increment, initial orbital elements, sun vectors, and magnetic field vectors both in the body and inertial system. The satellite used is a typical nanosatellite with the $I_{x}=0.1430 \mathrm{kgm}^{-3}, I_{y}=0.1020 \mathrm{kgm}^{-3}$, and $I_{z}=0.0031 \mathrm{kgm}^{-3}$. Attitude control is achieved by using a closed-loop feedback system using PID and Direct torque control. The two attitude control modes which are detumbling and stabilization mode are simulated.

Initially, the satellite is simulated from the time it is ejected and experiencing tumbling for several seconds prior to the activation of detumbling mode. Most of the micro, nano and pico-satellites are using B-dot algorithm [21]. As expected, during the detumbling mode satellite's Euler angles increases exponentially due to the absence of any control torque to inhibit their motion. The overall attitude control mode is simulated for the desired angle at ideal, normal and DTC and the simulation results are given in Figure 2, 3, and 4.

During the stabilization mode, roll angle and yaw angle still experiences the increment slowly but not exponentially. The gravity gradient torque did successfully reduce the motion of the satellite. The pitch angle is completely stabilized since its motion independent of roll and yaw pitch angular rate. The slight increase in roll and yaw angle is due to the influence of their angular rate on each other based on the dynamic equations of motion (11), (12) and (13).

Finally, the overall ADCS system is simulated using a PID controller to compute the input torque demand from the attitude error and actuator responsible to generate the actual torque based on the demand torque. The simulation of the overall attitude control mode with the ideal condition is shown in Figure 4. In an ideal condition, we assumed that the actuator exactly produces the demand torque for the desired Euler angle of 1.5 radians about the roll, pitch, and yaw angle. Using the Matlab auto-tuning for the PID controller, the best response for the closed-loop system is achieved. The pitch angle shows the fastest response followed by the roll and yaw. The pitch angle is fast because of the complete stabilization during the stabilization mode and due to the fact that its motion is independent of roll and yaw motion. The roll and yaw reach are the desired angle next since its response depends on each other. The yaw angular rate negatively influences the roll angle based on dynamic equations of the satellite. Yaw angular rate generates a high amount of positive torque to counter the motion about the roll.

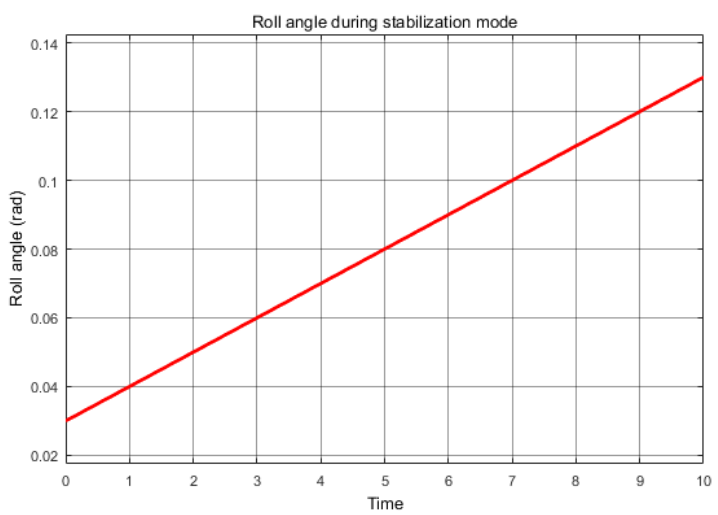

Figure 1.1: Roll angle during stabilization mode

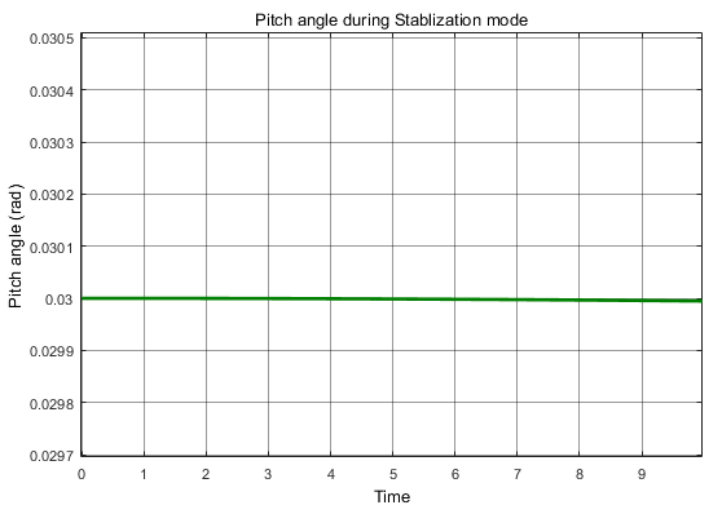

Figure 1.2: Pitch angle during stabilization mode

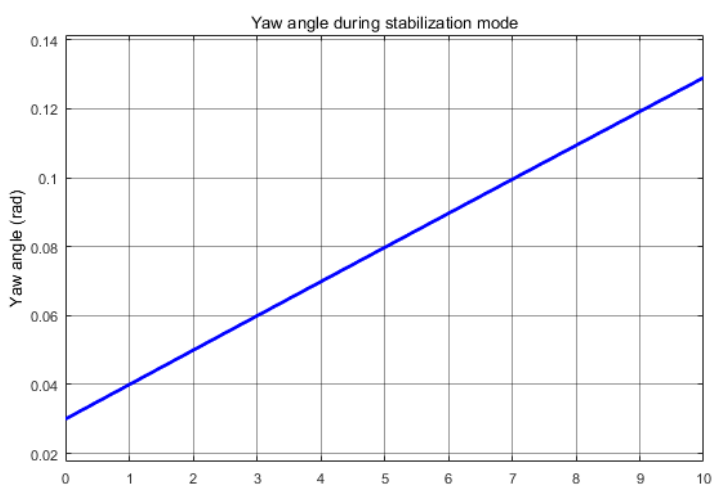

Figure 1.3: Yaw angle during stabilization mode 


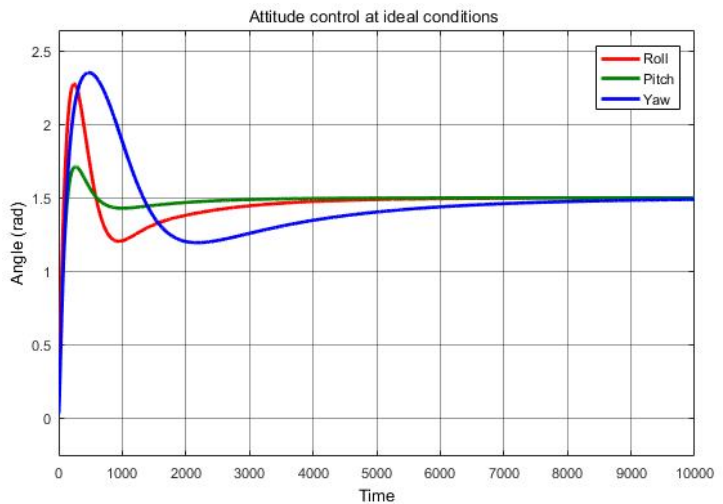

Figure 2: Overall attitude control for the desired angle $(1.5,1.5,1.5)$ radians at ideal conditions.

Hence, the roll angle achieves its desired response faster than yaw while yaw is slower due to the positive influence of the roll angular rate on the motion of the yaw angle. The roll angular rate generates a high amount of negative torque that supports the motion about the yaw angle. Thus, attitude control about yaw angle requires a high amount of torque from the Direct Torque Control. This causes the generation of the desired response as shown in Figure.3.

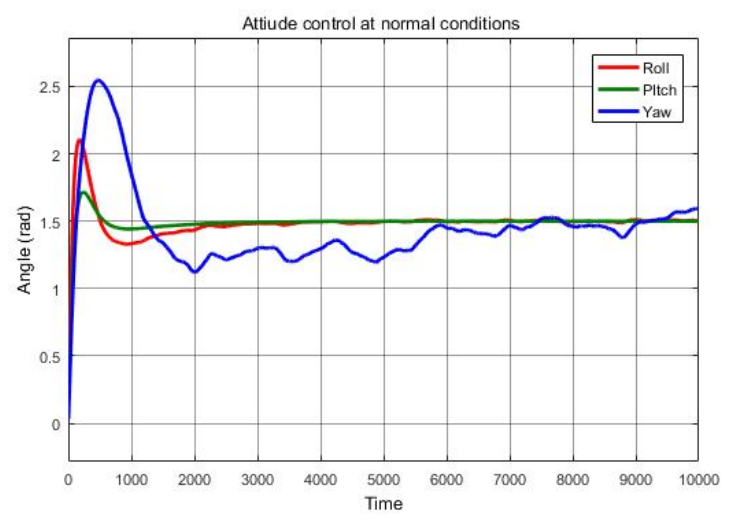

Figure 3: Overall attitude control for the desired angle (1.5, $1.5,1.5)$ radians in normal conditions.

Then overall ADCS is simulated using a closed-loop feedback system again with actuator under normal conditions. The torque generated not exactly equal to the demand. The simulation is shown in Figure.4. The overall system is successfully showing some error in the torque generated by the actuator which causes the inaccuracies in the satellite's attitude.

At last overall ADCS system is simulated again with the implementation of Direct Torque Control on the actuator. The results are shown in Figure.6. The ADCS system with Direct Torque Control on the actuator successfully corrects the actual torque generated by the actuator. The implementation of Direct Torque Control on the actuator effectively control the stator flux using switching table and inverter of the actuator and can be one of the efficient ways to achieve a good attitude control on a satellite.

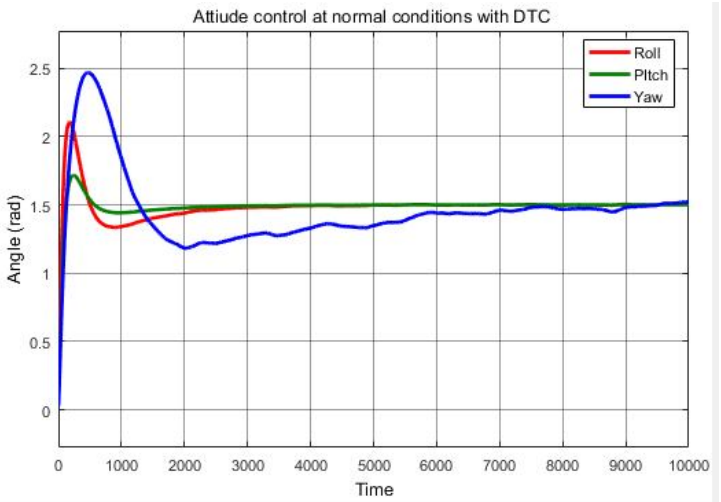

Figure 4: Overall attitude control for the desired angle $(1.5,1.5,1.5)$ radians at normal conditions with DTC.

\section{CONCLUSION}

The goal of this paper is developed an ADCS system of a satellite using Direct Torque Control. The Direct Torque Control is used as a component to effectively control the torque generated by the actuator to produce the demand torque accurately. Based on the results, it is proven that DTC can be one of the efficient ways to control the attitude of the satellite in space due to the ability of DTC to control the correct the torque generated.

Future developments involve the advancement of the current ADCS model. Currently, the ADCS environmental model is run with simple orbit propagator. Thus, in the future, the system will be updated with Simplified Perturbation Model, SGP4 using Two Line Element set and time since epoch for the orbital model. Attitude determination mode will be improved with the Extended Kalman filter and hardware development for the real satellite will be carried out to check for complete effectiveness of the system in a real environment.

\section{ACKNOWLEDGMENT}

Funding by the Ministry of Higher Education Malaysia under the research grant FRGS/1/2019/TK04/UKM/02/10 is gratefully acknowledged. The authors would also like to thank H. F. Abdul Wahab for providing the necessary material needed to complete this project.

\section{REFERENCES}

[1] Bak, T., Wisniewski, R., \& Blanke, M., 1996. Autonomous Attitude Determination and Control System for the orsted Satellite. Proceedings of the 1996 IEEE Aerospace Applications Conference, Snowmass at Aspen, Colorado February 3-10 1996. 1-12.

[2] Christoffel J. G., 2014. Attitude Determination and Control System or EyasSAT for Hardware in the Loop Application. Degree Thesis. Stellenbosch University.

[3] Christopher D. H., 2003. Spacecraft Attitude Dynamics and Control, Chapter 4. 
http://www.aoe.vt.edu/ cdhall/courses/aoe4140/attde.p df.

[4] Cortiella, A., Vidal, D., Jané, J., Juan, E., Olivé, R., Amézaga, Joan Francesc Munoz, Pol Via Hugo Carreno-Luengo and Adriano Camps. 2016. 3CAT-2: Attitude Determination and Control system for a GNSS-R Earth Observation 6U Cubesat mission. European Journal of Remote Sensing, 49 (November), 759-776. https://doi.org/10.5721/EuJRS20164940

[5] De Oliveira, G. F., Ishihara, J. Y., Borges, R. A., Ferreira, H. C., Kulabukhov, A. M., Larin, V. A., \& Belikov, V. V. 2013. A Low-Cost Attitude Determination and Control System for the UYS-1 nanosatellite. IEEE Aerospace Conference (2013), 1-7.

[6] Chessab, M. M., 2013. Gravity Gradient Stabilization of Nano Satellite Using Fuzzy Logic Controller, Thesis Master, University of Kufa.

[7] Chessab, M. M., 2014. Attitude Determination and Control System Design of Kufasat. International Journal of Current Engineering and Technology. (4), 1-9.

[8] Sidi, M.J., 1997. Spacecraft Dynamics and Control: A Practical Engineering Approach: Cambridge University Press.

[9] Ni, S., \& Zhang, C. 2011. Attitude Determination of Nano Satellite based on Gyroscope, Sun-sensor, and magnetometer. Procedia Engineering, 15, 959-963, $1-5$. https://doi.org/10.1016/j.proeng.2011.08.177

[10] Sabatini, R., \& Kaharkar, A. 2013. Carrier-phase GNSS Attitude Determination and Control for Small Unmanned Aerial Vehicle Applications. Journal of Aeronautic \& Aerospace, 2(11), 297-322, 1-23.

[11] Wahab, H. F. A., Sanusi, H., 2008. Simulink Model of Direct Torque Control of Induction Machine. American Journal of Applied Sciences, 5 (8)(8), 1-8.

[12] Kaplan, M. H., 1976. Modern Spacecraft Dynamics and Control, Vol. 1. New York: John Wiley and Sons, Inc., p. 427.

[13] Shuster, M. D., and Oh, S., 1981. Three-axis attitude determination from vector observations. Journal of Guidance, Control, and Dynamics, Vol. 4, 70-77. https://doi.org/10.2514/3.19717

[14] Mehrjardi, M. F., Sanusi, H., Ali, M., Alauddin, M., and Taher, M. A., 2014. PD controller for three-axis satellite attitude control using discrete

[15] Kalman Filter. In Proceedings of the 2014 International Conference on Computer, Communications, and Control Technology (I4CT), 83-85.

[16] Mehrjardi, M. F., Sanusi, H., Ali, M., Alauddin, M., and Taher, M. A., 2015. Proportional derivative controller using discrete Kalman filter estimation method for spacecraft attitude control. Applied Mechanics and Materials, 923-926.

[17] Markley, F. L., and Mortari, D., 2000. Quaternion attitude estimation using vector observations. Journal of the Astronautical Sciences, Vol. 48, 359-380.

[18] Mezher, L.S., 2019. Position Control for Dynamic DC MOTOR with Robust PID Controller using MATLAB.
International Journal of Advanced Trends in Computer Science and Engineering, 8( 3), May - June 2019, 936 942

https://doi.org/10.30534/ijatcse/2019/92832019

[19] Africa, A. D. M. et al., 2019. A Comprehensive Study of the Functions and Operations of Control Systems. International Journal of Advanced Trends in Computer Science and Engineering, 8(3), May - June 2019, 922926

https://doi.org/10.30534/ijatcse/2019/89832019

[20] Bar-Itzhack, I. Y., 2000. New method for extracting the quaternion from a rotation matrix. Journal of Guidance, Control, and Dynamics, Vol. 23, 1085-1087.

[21] Lovera, Marco. "Magnetic satellite detumbling: The b-dot algorithm revisited." 2015 American Control Conference (ACC) (2015): 1867-1872. https://doi.org/10.1109/ACC.2015.7171005 